\section{Qual deficiência? Perícia médica e assistência social no Brasil}

\author{
Which disability? Medical evaluation and social \\ assistance in Brazil
}

\author{
1 Universidade de Brasília, \\ Brasília, Brasil. \\ 2 International Poverty \\ Center/United Nations \\ Development Programme, \\ Brasília, Brasil. \\ Correspondência \\ D. Diniz \\ Universidade de Brasília. \\ C. P. 8011 , Brasília, DF \\ 70673-970, Brasil. \\ anis@anis.org.br
}

\begin{abstract}
This article examines the concept of disability adopted by the most important cash transfer program targeting the disabled population in Brazil, the Continuous Cash Benefit (Beneficio de Prestação Continuada-BPC). The study compares the eligibility criteria established by law and the criteria used by medical examiners in the beneficiary selection process. The data are from a sample survey of $16 \%$ of the medical examiners working in the program. The questionnaire aims to assess the instructions, forms, and procedures for selecting disabled beneficiaries. The results show a discrepancy between the formal program criteria and actual practice by the examiners, suggesting an expanded concept of disability aimed at including beneficiaries with genetic, chronic, and severe infectious diseases.
\end{abstract}

Social Assistance; Disabled Persons; Social Security
Debora Diniz 1

Flávia Squinca 1

Marcelo Medeiros 2

\section{Introdução}

O Benefício de Prestação Continuada (BPC), em funcionamento no Brasil desde 1993, é uma transferência incondicional de renda para idosos ou pessoas com deficiência, extremamente pobres. As transferências são concedidas a pessoas idosas ou pessoas com deficiência grave, cuja renda familiar per capita seja inferior a um quarto de salário mínimo ${ }^{1}$. O valor da transferência é equivalente a um salário mínimo mensal. As transferências são independentes de contribuições prévias para o sistema de seguridade social e não são condicionadas a qualquer contrapartida. Todas as pessoas, extremamente pobres acima de 65 anos 2 2 deficientes ou não, são elegíveis ao benefício. No caso dos deficientes não-idosos, apenas aqueles extremamente pobres classificados como possuindo deficiência grave que incapacita para a vida independente e para o trabalho podem receber o BPC. Peritos do BPC realizam testes para avaliar tanto a situação social quanto a condição de deficiência. O sistema de transferências prevê reavaliações sistemáticas a cada dois anos para verificar a persistência dessas condições ${ }^{3}$.

O gasto com transferências representa uma fração pequena (9\%) do montante total transferido pelo sistema previdenciário de regime geral, que inclui os programas de assistência social. O programa conta com cerca de 2,1 milhões de beneficiários, dos quais, pelo menos, 1,1 milhão 
são pessoas com deficiência 4 . As informações de caráter demográfico sobre a concessão de benefícios para pessoas deficientes no ano de 2004, obtidas no processo de cadastramento dos novos beneficiários processado pela Empresa de Tecnologia e Informações da Previdência Social (DATAPREV), indicam que grande parte das concessões por deficiência ocorre entre crianças e jovens 5 . Quarenta e dois por cento dos benefícios foram concedidos a pessoas em idades entre $0 \mathrm{e}$ 24 anos, sendo boa parte deles concentrados nas idades mais jovens. A população de 25 a 45 anos representa $29 \%$ das novas concessões; e a população de 46 a 64 anos, uma fração igual, 29\% 4 . Entre a população idosa, é importante lembrar que o BPC é concedido a pessoas com deficiências, mas também a pessoas idosas em situação de pobreza. Ou seja, é possível que, entre a parcela de idosos no BPC, exista um número considerável de idosos deficientes, mas que se encontram classificados na categoria idosos e não deficientes.

O objetivo deste artigo é analisar o conceito de deficiência adotado pelo BPC. A hipótese de pesquisa foi de que há uma intensa controvérsia entre diferentes modelos de compreensão da deficiência, em especial entre o modelo social e o modelo médico da deficiência 6 . Um comentário referente à terminologia sobre deficiência merece ser destacado. Há uma controvérsia sobre a terminologia correta a ser utilizada para referirse às pessoas e populações com deficiência 7,8 . Por julgar que essa controvérsia não é central para os objetivos deste artigo, utilizamos indiscriminadamente os termos pessoa deficiente, deficiente, pessoas com deficiência e, eventualmente, pessoas portadoras de deficiência.

\section{Metodologia}

O estudo contrasta os critérios de seleção regulamentados pelo programa aos critérios efetivamente utilizados pelos médicos peritos encarregados de avaliar e selecionar os beneficiários do BPC. O contraste entre norma e prática é acompanhado de uma análise dos principais motivos que levam a divergência entre ambos e a consistência das decisões práticas com os objetivos últimos das políticas de assistência social no Brasil. Os dados levantados para este artigo provêm das seguintes fontes de informação: (1) um levantamento baseado em questionários aplicados a uma amostra incidental de médicos peritos de todo o país que realizam parte da seleção dos beneficiários e (2) levantamentos de toda a legislação e todos os regulamentos relacionados ao programa desde sua implementação.
À época do levantamento, o número total de médicos peritos no país era de 3.300. A única maneira viável de contatá-los foi por intermédio da listagem dos membros da Associação Nacional dos Médicos Peritos, que congrega cerca de $90 \%$ dos peritos brasileiros 9 . Para a realização do levantamento, foi distribuído um questionário eletrônico a todos os médicos peritos listados, e os questionários retornados resultaram em uma amostra incidental de 16\% do total de 3.019 médicos peritos $(n=483)$. Para referência, caso se tratasse de uma amostragem aleatória simples, o erro amostral seria de, aproximadamente, 0,04.

O questionário consistiu de seis perguntas, sendo que duas testavam valores por meio de escalas de julgamento. Essas perguntas levantaram informações de caráter demográfico e avaliaram a qualidade das instruções que os peritos recebem sobre como conduzir o processo de avaliação, a qualidade dos formulários criados pelo Instituto Nacional de Seguro Social (INSS) e utilizados nesse processo e os procedimentos adotados, na prática, pelos médicos para implementar os critérios de elegibilidade determinados legalmente para a concessão do BPC para as pessoas deficientes. A estrutura do questionário girou em torno da análise dos mecanismos de seleção de beneficiários, uma vez que esse mecanismo é um dos eixos centrais de execução de qualquer política focalizada.

\section{O significado da deficiência}

Como no caso da saúde, da educação ou até mesmo da pobreza, há diferentes definições para a deficiência. Regra geral, deficiência pressupõe a existência de variações de algumas habilidades que sejam qualificadas como restrições ou lesões. O que inexiste, no entanto, é um consenso sobre quais variações de habilidades e funcionalidades caracterizariam deficiências. Há pessoas com lesões que não experimentam a deficiência, assim como existem pessoas com expectativa de lesões que se consideram deficientes 10,11 . Traçar a fronteira conceitual entre essas diversas expressões da diversidade humana é um exercício intelectual na fronteira de diferentes saberes, em especial entre o conhecimento médico e as ciências sociais. Essa variedade de interpretações e experiências em torno do corpo e da relação deste com o ambiente social perpassam grande parte das discussões contemporâneas sobre deficiência e justiça social 12,13,14,15. E não é por acaso que essa é também uma das questões mais controversas para a garantia do acesso ao BPC.

Uma das saídas para solucionar essa controvérsia seria listar quais variações de habilidades 
deveriam caracterizar-se como deficiência para a identificação de beneficiários. Esse é um argumento intensamente discutido nos circuitos de especialistas do BPC, seja entre representantes governamentais, parlamentares, movimentos sociais e médicos peritos. Caso fosse possível classificar e qualificar as habilidades, essa seria uma saída normativa que facilitaria o processo de seleção e inclusão no programa, mas que ignoraria a complexidade da relação entre as habilidades, as funcionalidades e o contexto social em que vive cada pessoa. A idéia de deficiência é freqüentemente relacionada a limitações naquilo que se considera como habilidades básicas para a vida social 14,15. Não é fácil determinar quais são essas habilidades, muito embora grande parte do debate as relacione à mobilidade, ao uso dos sentidos, à comunicação, à interação social e à cognição. Uma outra condição para caracterizar uma variação de habilidade como deficiência é que essa se expresse no corpo como um estado permanente ou de longa duração. Dificilmente pessoas que encontram dificuldades de leitura porque são analfabetas e não tiveram acesso à escola serão consideradas deficientes, mas uma pessoa cega privada de braile e exposta à escrita gráfica seria considerada deficiente 10,11.

As habilidades para executar diferentes tarefas não são distribuídas igualmente na população. Se a distribuição de habilidades básicas para a caracterização da deficiência for vista em um continuum, é possível reconhecer a existência de desigualdades com respeito à intensidade das variações de deficiência em uma determinada população. Definir a variação da habilidade a ser considerada como uma lesão ou como uma restrição é, fundamentalmente, um julgamento de valor 16. Isso não significa que, ao afastar o debate sobre a deficiência de um campo essencialmente médico-normativo e aproximá-lo de um debate sobre quais diferenças de habilidades justificariam ações de reparação de desigualdade, perderemos os critérios objetivos da perícia médica para a inclusão de uma pessoa no BPC. O fato é que, apesar de a maioria das definições de deficiência basear-se em variações corporais qualificadas como lesões, os dois conceitos (lesões e deficiência) não são sinônimos 8,17.

Na tentativa de dirimir parte dessa controvérsia conceitual, a Organização Mundial da Saúde (OMS) publicou uma revisão da classificação terminológica sobre deficiência em 2001 11. Segundo a Classificação Internacional de Funcionalidade, Incapacidade e Saúde (CIF), "as deficiências são problemas nas funções ou nas estruturas do corpo com um desvio importante ou perda" 11 (p. 21). Para a CIF, a funcionalidade e a incapacidade de um indivíduo são resultado da interação entre os estados de saúde e o meio ambiente, em que a deficiência é resultado de uma interação complexa das pessoas com a sociedade 18 .

Em um ambiente hostil à diversidade corporal, é possível imaginar uma pessoa com restrições leves de habilidades que experimente a deficiência de forma severa. A mesma restrição de habilidade, em um ambiente receptivo à diversidade, pode não levar à experiência da deficiência 10,11. Essa relação complexa entre corpo, habilidades e sociedade aponta para o fato de somente ser possível avaliar adequadamente o nível de deficiência elegível ao BPC se informações sobre o ambiente forem seriamente incorporadas ao protocolo de perícia. Não seria equivocado, portanto, considerar diferentes definições de deficiência para os programas sociais. Essas definições partiriam das variáveis de habilidades básicas, lesões e determinantes sociais, mas não pressuporiam um caráter absoluto, mas sim uma interação complexa entre elas.

Para fins de concessão do BPC, o Decreto $n^{\circ}$. 1.774, de 1995, estabelece a pessoa portadora de deficiência como sendo "aquela incapacitada para a vida independente e para o trabalho, em razão de anomalias ou lesões irreversíveis de natureza hereditária, congênitas ou adquiridas, que impeçam o desempenho das atividades da vida diária e do trabalho" 19. A inspiração para essa definição foi o modelo médico da deficiência, um conjunto de teorias e práticas assistenciais em saúde que pressupõem uma relação de causalidade entre a lesão e a experiência da deficiência 20 . Sob essa perspectiva, ainda hegemônica no debate sobre deficiência no Brasil, a deficiência seria a expressão de uma limitação corporal do indivíduo para interagir socialmente. Para a comprovação da deficiência, a pessoa é submetida a uma perícia médica realizada pelo INSS, o que, na prática, atrela a definição de deficiência a avaliações médicas ad hoc.

Uma análise mais cuidadosa do conceito de incapacidade permanente sugere ser esse uma tentativa de demarcar as fronteiras entre deficiência e doença, em um contexto discursivo em que deficiência, diferentemente de doença, seria um estado refratário ao tratamento ou mesmo à cura. Essa diferença entre estado (deficiência) e condição (doença) seria uma das explicações possíveis para a ênfase no modelo médico da deficiência nos mecanismos da perícia e nos critérios de inclusão para o benefício. A sobrevalorização do discurso médico em detrimento das variáveis sociológicas da deficiência não parece estar em harmonia com os princípios igualitários que fundamentam o programa. Ao adotar a definição de incapacidades permanentes, várias condições que poderiam ser entendidas como 
deficiências para o BPC são automaticamente excluídas.

A lista de incapacidades permanentes elegíveis foi definida por um decreto em 1999 e excluiu algumas incapacidades tradicionalmente associadas à deficiência ${ }^{21,22}$. Basicamente, a lista de incapacidades reduziu-se a limitações visuais e auditivas graves, tipos de paralisia física de origem neurológica, ausência de algum membro e uma lista vaga de incapacidades mentais manifestadas antes dos 18 anos. Se as definições fossem estritamente seguidas, pessoas com problemas neurológicos degenerativos, artrite, limitações circulatórias graves, HIV/AIDS sintomática, doenças renais e esquizofrenia intermitente, só para citar alguns, não seriam elegíveis ao BPC, mesmo quando essas condições impedissem o trabalho, causassem dependência para os cuidados de atividades de vida diária e resultassem em extrema pobreza.

\section{A perícia médica}

Os médicos peritos responsáveis pelos exames para o BPC já consideram algumas doenças crônicas como uma condição elegível ao benefício. A pesquisa com os peritos do INSS mostrou que $82 \%$ dos médicos peritos consideram uma pessoa em estágio avançado de infecção de HIV/AIDS e $46 \%$ consideram que uma pessoa com quadro de artrose grave como elegíveis ao BPC. Nesses dois casos, o fator determinante para a inclusão no programa não seria apenas a condição do HIV/ AIDS ou da artrose, mas o fato de a primeira já estar em estágio avançado de infecção e a segunda a gravidade da artrose. Quando questionados sobre uma pessoa com artrose em crises regulares de dor, apenas $2 \%$ dos médicos peritos a identificariam como elegível ao BPC. O desafio que esses resultados levantam não é o da inclusão de doenças crônicas para a elegibilidade ao BPC, mas sim sobre como definir critérios claros para que cada pessoa seja tratada do mesmo modo no processo de seleção.

Mas, no que se refere às incapacidades elegíveis, se as regras fossem rigorosamente cumpridas, cada incapacidade deveria ser considerada isoladamente, o que também não seria coerente com os princípios que fundamentam a existência do BPC. O desenho do programa não considera, por exemplo, a possibilidade de que várias incapacidades moderadas possam gerar uma experiência severa de deficiência. A idéia de pessoa deficiente elegível ao BPC seria aquela com uma incapacidade grave ou extremamente debilitante. Essa compreensão estreita de deficiência poderia resultar numa exclusão sistemática inde- sejada de parte da população com as chamadas “deficiências múltiplas", mas que não sejam classificadas isoladamente como severas.

Apesar das regras do programa, há evidências de que novas interpretações guiem a prática de concessão do benefício. Os resultados da pesquisa com os médicos peritos mostram que uma pessoa com insuficiência renal crônica seria considerada elegível para $28 \%$ dos médicos. Se essa insuficiência fosse combinada com diabetes, a taxa de aprovação dobraria para 57\% dos examinadores, e isso não seria causado pela presença de diabetes, mas pela combinação das duas condições. A intensidade da condição também é uma variável para determinar a elegibilidade: uma criança com anemia falciforme, uma doença genética prevalente em afro-descendentes, com crises regulares de dor seria elegível ao BPC para $14 \%$ dos médicos peritos. Mas, se essa mesma criança já tiver apresentado dois acidentes vasculares cerebrais, o índice de aprovação seria de $90 \%$. Ou seja, os médicos peritos já ponderam a existência de incapacidades múltiplas, de graus variados de intensidade da condição ou de fatores agravantes, apesar de a legislação ser omissa em relação a isso.

O BPC usa a definição de deficiência mental proposta pelo Decreto $n^{o} .3 .298$ 21, de 1999, que regulamentou a Política Nacional para a Integração da Pessoa Portadora de Deficiência, a qual determina que deficiência mental é o "funcionamento intelectual significativamente inferior à média, com manifestação antes dos 18 anos $e$ limitações associadas a duas ou mais áreas de habilidades adaptativas, tais como comunicação, cuidado pessoal, habilidades sociais, utilização dos recursos da comunidade, saúde e segurança, habilidades acadêmicas, lazer e trabalho" 21. Não restam dúvidas do quanto é difícil definir a deficiência mental por critérios objetivos e universais. Mais do que qualquer outra expressão da diversidade humana, a deficiência mental pressupõe uma ampla revisão do modelo médico da deficiência, em especial dos conceitos de normal e patológico 23 . No entanto, pelo menos aspectos das definições atuais relativas à deficiência mental distanciam a legislação dos objetivos da política.

Assim como nos estudos sobre pobreza em que se considera inadequado assumir uma linha de pobreza em função da renda média da população, não é a melhor estratégia basear-se no desempenho intelectual médio de uma população como referência para o BPC. Mesmo se o conceito de "significativamente inferior à média" pudesse ser precisado - talvez por meio de algum teste padronizado -, a distância entre as médias não seria o método mais apropriado para defi- 
nir-se parâmetros para habilidades e capacidades cognitivas e mentais. Além dessa dificuldade inerente à mensuração da competência, é comum confundir-se "média" com "normalidade". Normalidade é antes um valor moral, um julgamento sobre tipos humanos ideais e padrões de bem viver que mesmo um conceito neutro que permita uma avaliação médica de elegibilidade ao BPC 24. Não é possível afirmar se essa confusão entre "normal" e "média" esteve por trás do Decreto $n^{o} .3 .298$ 21, muito embora seja essa uma transposição conceitual muito comum ao modelo médico da deficiência 22 . O fato é que se o objetivo do BPC é assistir pessoas incapazes de viver independentemente e de trabalhar, então os critérios avaliativos de desempenho mínimo deveriam ser estabelecidos tendo essas duas variáveis em consideração.

\section{Os critérios de elegibilidade}

Para uma pessoa ser elegível ao BPC, um dos critérios é que as restrições cognitivas ou mentais se manifestem antes dos 18 anos. Esse critério foi contestado pela maioria dos médicos peritos entrevistados, pois 55\% deles consideram a idade uma variável de baixa importância para a avaliação de uma pessoa ao BPC. A exigência de que a condição se manifeste até um limite de idade impede, desnecessariamente, que pessoas com deficiências graves fora dessa faixa etária se beneficiem do programa. Como parte do sistema de assistência social, o BPC dificilmente poderia justificar a exclusão de pessoas que desenvolvam suas limitações intelectuais na maturidade ou na velhice. A não ser que se considere que a deficiência mental apresenta características particulares para a inclusão de uma pessoa no programa somente em uma determinada faixa etária, uma simples analogia com outras condições, como a deficiência física, mostra o quanto a exclusão de pessoas com deficiência mental acima de 18 anos infringe o princípio do igual tratamento. Como não há argumentos que justifiquem essa exclusão, o acesso a direitos sociais de pessoas com doenças degenerativas, condições psiquiátricas que só se desenvolvem depois da maturidade ou perdas de funções neurológicas devido a doenças infecciosas, não deveria ser impedido pelo critério etário.

Para ser elegível ao BPC, é preciso ainda que a pessoa seja "incapacitada para o trabalho e para a vida independente", e o desafio é exatamente sobre como definir essas duas categorias. Não há critérios objetivos e uniformes para defini-las, o que termina por transferir para os médicos peritos a responsabilidade pelo julgamento final. $\mathrm{O}$ desafio está tanto na dificuldade de definir trabalho e independência, categorias repletas de valor e avaliações sobre o bem viver, mas também porque muitas das restrições para o trabalho ou para a vida independente não estão no indivíduo, mas na interação com o meio no qual a pessoa deficiente vive ${ }^{8}$. Pouquíssimas são as pessoas deficientes incapacitadas de forma plena para o trabalho. O que há são muitas pessoas com restrições moderadas de habilidades que enfrentam graves restrições no mercado de trabalho em conseqüência de variáveis não mensuráveis, tais como discriminação, preconceito ou barreiras sociais de outra ordem 25 . Nesse sentido, a categoria "incapacidade para o trabalho" exige tanto um conhecimento clínico para o diagnóstico de habilidades e capacidades corporais, mas também um extenso e cuidadoso diagnóstico do universo social e do trabalho em que a pessoa deficiente vive.

Pessoas deficientes menores de 16 anos são automaticamente consideradas "incapazes para o trabalho". Essa é uma das razões que explica a maior proporção da população menor que 18 anos entre os beneficiários, 35\% do total 5. Mas essa concentração de beneficiários entre os jovens pode ser analisada sob duas perspectivas. A primeira perspectiva considera uma maior prevalência à pobreza nas famílias com crianças deficientes. As crianças e adolescentes seriam os principais beneficiários, pois é nessa população que está a maior parcela de deficientes com graves restrições de habilidades ocorrendo simultaneamente a uma situação de pobreza. A segunda perspectiva, apesar de não ignorar uma possível prevalência da pobreza entre jovens deficientes, aponta para a sobrevalorização do critério "trabalho" no processo de avaliação. Ou seja, potenciais beneficiários adultos seriam excluídos do programa por serem considerados aptos para o trabalho, muito embora os critérios de avaliação não contemplem a complexidade do fenômeno da deficiência, mas essencialmente as restrições clínicas corporais. Ou seja, quando a categoria "trabalho" não é considerada, as chances de inclusão de uma pessoa no programa crescem, e isso não apenas pelo recorte etário.

Há ainda outro desafio na pouca especificidade do conceito "incapacidade para o trabalho". Uma longa tradição de estudos sobre deficiência já demonstrou o quanto as pessoas deficientes foram submetidas a discursos moralizadores pautados em premissas religiosas ou na compaixão individual, o que redefiniu as pessoas deficientes ora como aquelas "pobres merecedoras da ajuda", ora como aquelas "pobres, porém que não desejam trabalhar" 26. A assistência social é uma expressão de um direito constitucional que pro- 
tege pessoas em situação de desvantagem 1,27. As pessoas deficientes em processo de seleção para a inclusão no BPC não necessitam submeter-se a julgamentos sobre o significado da deficiência em suas vidas, tampouco a um julgamento moral sobre suas motivações para o trabalho.

A população de deficientes elegível ao BPC é um grupo de pessoas em extrema pobreza, ou seja, chega a ser desnecessário testar capacidades em uma população que tem a busca por mínimos sociais como um incentivo para o trabalho 28 . Um projeto de lei (PL no. 4.005/2001; Altera o Art. 20 da Lei $n^{\circ}$. 8.742, de 7 de dezembro de 1993, que Dispõe Sobre o Benefício de Prestação Continuada da Assistência Social aos Idosos e aos Portadores de Deficiência Carentes), em tramitação no Congresso Nacional, busca solucionar essa tensão moral, ao sugerir uma mudança na definição de quem seja a pessoa deficiente: "pessoas que sofrem de limitações físicas, mentais ou emocionais que dificultam seu trabalho remunerado ou sobrevivência". Apesar de essa nova definição ser ainda vaga e permitir ambigüidades interpretativas, ela representa tanto uma tentativa de solucionar a imprecisão da variável "incapacidade para o trabalho", mas também é uma tentativa de incorporar a relação entre indivíduo e meio social.

O Brasil, ao ratificar a Convenção Interamericana para a Eliminação de Todas as Formas de Discriminação Contra as Pessoas Portadoras de Deficiência, reconheceu o seu conteúdo com força de lei ${ }^{29}$. Baseada nessa convenção, uma ação civil pública bem-sucedida se opôs ao uso do critério "incapacidade para o trabalho e para a vida independente" pelo BPC, criando uma interpretação alternativa àquela proposta pelo programa. O caminho do Judiciário pode implicar em duas alternativas judiciais futuras. A primeira que essa nova interpretação ganhe força e passe a ser requerida em processos judiciais de revisão para a inclusão no BPC de forma crescente. Gradualmente, poderá ser uma interpretação também utilizada pelos médicos peritos para evitar ações de revisão judiciais com garantias de sucesso. A segunda alternativa é que essa interpretação seja vencida também judicialmente, seja por uma ação do Estado ou também pelo Ministério Público Federal, exigindo ou uma adequação legal pelo Executivo ou o cumprimento da interpretação oficial do programa.

O instrumento que avalia as variáveis do trabalho e da vida independente é um questionário padronizado e utilizado por todos os médicos peritos do país. Desde a implementação, o questionário já sofreu modificações por ordens e resoluções internas ao INSS, mas ainda é objeto de discussão entre os profissionais que o utilizam para selecionar os beneficiários. A pesquisa com os médicos peritos do INSS mostrou que $47 \%$ deles consideram o questionário de avaliação ineficiente ou menos que eficiente, e somente $5 \%$ acreditam que o questionário é muito eficiente para o processo de seleção. Essa baixa taxa de confiança no instrumento deve ser entendida para além de uma simples ineficácia do questionário: pode ser um indicador do quanto a mensuração das variáveis trabalho e vida independente é complexa e pouco permeável a um instrumento nos termos do atualmente em vigor.

Mas o resultado dessa baixa confiança tem também um efeito imediato e que impõe riscos à elegibilidade ao programa: os médicos peritos podem solucionar essa ineficiência do questionário incorporando outros mecanismos avaliativos à perícia. Caso pesquisas futuras comprovem essa saída personalista à ineficiência alegada do questionário, ela deve ser considerada problemática para o programa. Quanto mais padronizada for a seleção, maiores as chances de objetividade no acesso ao programa e menores os riscos de idiossincrasias pessoais dos avaliadores interferirem na elegibilidade.

As ações judiciais, as ações civis públicas, ou mesmo as novas interpretações adotadas pelos médicos peritos são, por um lado, a expressão de um processo contínuo de revisão de qualquer política pública e de garantias de direitos individuais, mas também podem representar ameaças para alguns dos fundamentos éticos do BPC, como a impessoalidade. Certamente essas são estratégias que solucionam, em curto prazo, entraves do programa, dificuldades dos instrumentos de seleção, ou mesmo atualizam o debate político em consonância com novas demandas e interpretações sobre a deficiência, mas qualquer decisão de caráter discricionário provoca sempre um impasse para a continuidade da política pública. Segundo o levantamento, 59\% dos médicos peritos trabalham como examinadores do INSS para o BPC há menos de quatro anos e, apesar de suas qualificações técnicas, não receberam treinamento específico para a tarefa que lhes foi designada, em especial não foram apresentados à complexidade do fenômeno médico e sociológico da deficiência na sociedade brasileira. É nesse contexto desafiante de uma política de transferência de renda para uma população em situação de extrema pobreza, de um corpo de peritos que não recebeu treinamento específico e de variáveis complexas de serem mensuradas que o programa depende de um sistema uniforme de seleção que deve ser implementado e continuamente revisto. 


\section{Considerações finais}

Com o envelhecimento populacional, o tema da deficiência estará na pauta dos debates de políticas públicas no Brasil nos próximos anos. $\mathrm{O}$ envelhecimento populacional pode representar restrições de funcionalidades às pessoas, e se a sociedade não for sensível a esse processo, os idosos experimentarão a deficiência. Será nesse novo cenário populacional que o significado da deficiência assumirá um papel central para as políticas focalizadas nessa população. Cabe ainda frisar o papel dos movimentos sociais de pessoas deficientes e de doenças crônicas que provocam uma redefinição política das desvantagens experimentadas pelas lesões.

As divergências entre as decisões práticas dos médicos peritos e as regulamentações do BPC ocorrem para aproximar o programa dos objetivos das políticas de assistência. Nesse sentido, os médicos estão, na prática, corrigindo os erros da legislação ordinária e de normatização incompleta ou mesmo pouco específica no programa.
O alargamento da compreensão da deficiência pelos médicos peritos corresponde a um movimento internacional de não mais descrever a deficiência em termos estritamente médicos da lesão, mas como resultado de uma relação complexa entre lesões, desigualdades e meio ambiente.

Nesse movimento de revisão conceitual, doenças genéticas, crônicas e infecciosas passam, gradativamente, a serem classificadas como deficiência para fins de garantidas de direitos sociais básicos, como são os de assistência. O movimento de alargamento do conceito de deficiência pelos médicos peritos deve ser entendido como uma forma de garantir o acesso a mínimos sociais por uma população extremamente pobre e apartada do mercado de trabalho. Isso não significa, no entanto, que os médicos peritos estejam irresponsavelmente considerando doenças como deficiência, mas sim que, em harmonia como um movimento político muito amplo, eles desafiam o discurso tradicional sobre deficiência como sinônimo de um conjunto específico de lesões.

\section{Resumo}

Este artigo analisa o conceito de deficiência adotado pela principal política de transferência de renda para a população portadora de deficiência no Brasil, o Benefício de Prestação Continuada (BPC). O estudo contrasta os critérios de seleção regulamentados pelo programa aos critérios utilizados pelos médicos peritos encarregados de avaliar e selecionar os beneficiários do programa. Foi realizada uma pesquisa por amostra com 16\% do total de médicos peritos no Brasil. O objetivo do questionário foi avaliar a qualidade das instruções, dos formulários e dos procedimentos relativos à elegibilidade das pessoas deficientes ao benefício. Os resultados mostram que há uma divergência entre os critérios formais que regulamentam o BPC $e$ a prática pericial dos médicos que apontam para um alargamento do conceito de deficiência para a inclusão de beneficiários com doenças genéticas, crônicas e infecciosas graves.

Assistência Social; Pessoas Portadoras de Deficiências; Previdência Social

\section{Colaboradores}

Os três autores foram responsáveis por todas as fases de pesquisa, levantamento e tabulação de dados e redação do artigo.

\section{Agradecimentos}

A pesquisa que fundamentou este artigo foi financiada com recursos do Banco Mundial, administrados pela Cnotinfor, da Fundação Ford e do Conselho Nacional de Desenvolvimento Científico e Tecnológico (CNPq). Agradecemos à Associação Nacional dos Médicos Peritos por ter colaborado na fase de levantamento de dados e à leitura e aos comentários de Wederson Santos. 


\section{Referências}

1. Brasil. Lei no. 8.742. Dispõe sobre a Lei Orgânica da Assistência Social. Diário Oficial da União 1993; 8 dez.

2. Brasil. Lei ${ }^{\circ}$. 10.741. Dispõe sobre o Estatuto do Idoso e dá outras providências. Diário Oficial da União 2003; 1 out.

3. Ministério da Previdência Social e Assistência Social. Relatório do processo de revisão. Brasília: Ministério da Previdência Social e Assistência Social; 2002.

4. Ministério do Desenvolvimento Social e Combate à Fome. Benefício de prestação continuada. http://www.mds.gov.br/programas/rede-suas/ protecao-social-basica/beneficio-de-prestacaocontinuada-bpc (acessado em 10/Out/2005).

5. Empresa de Tecnologia e Informações da Previdência Social. Benefício de prestação continuada. http://www.dataprev.gov.br (acessado em 28/ Out/2005).

6. Empresa de Tecnologia e Informações da Previdência Social. Cash benefits to disabled persons in Brazil: an analysis of BPC. Brasília: Instituto de Pesquisa Econômica Aplicada; 2006.

7. Union of Physically Impaired Against Segregation. Fundamental principles of disability. London: Union of Physically Impaired Against Segregation; 1976.

8. Oliver M. The politics of disablement: critical texts in social work and the welfare state. London: MacMillan; 1990.

9. Associação Nacional dos Médicos Peritos. Estatística dos médicos peritos do INSS. http://www. perito.med.br (acessado em 01/Out/2005).

10. Organização Mundial da Saúde. Classificação internacional de funcionalidade, incapacidade e saúde. Brasília: Organização Mundial da Saúde; 2001.

11. Organização Mundial da Saúde. CIF: classificação internacional de funcionalidade, incapacidade e saúde. São Paulo: EdUSP; 2003.

12. Davis D. Choosing for disability. In: Davis D, editor. Genetic dilemmas: reproductive technology, parental choices and children's future. New York: Routledge; 2001. p. 49-67.

13. Wendell S. The rejected body: feminist philosophical reflections on disability. New York: Routledge; 1996.

14. Dworkin R. A virtude soberana: a teoria e a prática da igualdade. São Paulo: Editora Martins Fontes; 2005 .

15. Francis L P, Silvers A. American with disabilities: exploring implications of the law for individuals and institutions. New York: Routledge; 2000.
16. Barton L, Oliver M. Introduction: the birth of disability studies. In: Barton L, Oliver M, editors. Disability studies: past, present and future. Leeds: The Disability Press; 1997. p. ix-xiv.

17. Oliver M, Barnes C. Disabled people and social policy: from exclusion to inclusion. London: Longman; 1998.

18. Gleeson BJ. Disability studies: a historical materialist view. Disabil Soc 1997; 12:179-202.

19. Brasil. Decreto ${ }^{\circ}$. 1.744 , de 8 de dezembro, de 1995. Regulamenta o benefício de prestação continuada devido à pessoa portadora de deficiência e ao idoso, de que trata a Lei $n^{\circ}$. 8.742, de 7 de dezembro de 1993, e dá outras providências. Diário Oficial da União 1995; 8 dez.

20. Diniz D. O que é deficiência? São Paulo: Editora Brasiliense; 2007.

21. Brasil. Decreto $\mathrm{n}^{\circ}$. 3.298. Regulamenta a Lei $\mathrm{n}^{\circ}$. 7.853, de 24 de outubro de 1989, dispõe sobre a Política Nacional para a Integração da Pessoa Portadora de Deficiência, consolida as normas de proteção, e dá outras providências. Diário Oficial da União 1999; 20 dez.

22. Brasil. Lei $n^{\circ}$. 7.853. Dispõe sobre a Política Nacional para a Integração da Pessoa Portadora de Deficiência, consolida as normas de proteção, e dá outras providências. Diário Oficial da União 1989; 24 out.

23. Kittay E. Love's labor: essays on women, equality, and dependency. New York: Routledge; 1999.

24. Diniz D, Squinca F, Medeiros M. Deficiência, cuidado e justiça distributiva. In: Fontes M, Costa S, Squinca F, organizadores. Tópicos em bioética. Brasília: Editora Letras Livres; 2006. p. 82-94.

25. Abberley P. The concept of oppression and the development of a social theory of disability. Disabil Handicap Soc 1987; 2:5-19.

26. Oliver M, Barnes L. Disability studies today. Cambridge: Polity Press; 2002.

27. Boschetti I. Assistência social no Brasil: um direito entre originalidade e conservadorismo. Brasília: Grupo de Estudos e Pesquisas em Seguridade Social e Trabalho, Serviço Social, Universidade de Brasília; 2001.

28. Pereira P. A assistência social na perspectiva dos direitos - crítica aos padrões no Brasil. Brasília: Thesaurus Editora; 1996.

29. Brasil. Decreto no. 3.956. Convenção Interamericana para Eliminação de Todas as Formas de Discriminação Contra as Pessoas Portadoras de Deficiência. Diário Oficial da União 2001; 8 out.

Recebido em 05/Jul/2006

Versão final reapresentada em 06/Fev/2007 Aprovado em 19/Abr/2007 